\author{
Military Technical College \\ Kobry El-kobbah, \\ Cairo, Egypt
}

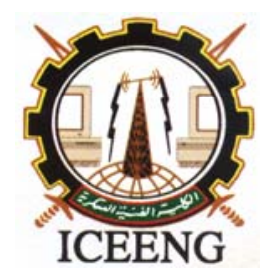

\title{
REGION OF INTEREST-BASED MEDICAL IMAGE COMPRESSION, WITH APPLICATION TO MRI BRAIN IMAGES
}

\author{
H. Aboelsoud M. , M. E Gadala*, A. S. Ragab ${ }^{*}$, and Abdalla S. Ahmed ${ }^{* *}$
}

\begin{abstract}
MRI Medical imaging produces human body pictures in digital form. Since these imaging modalities produce incredible amounts of data, therefore, compression is necessary for storage and communication purposes. Many current compression schemes provide a very high compression rate but with considerable loss of quality. On the other hand, in some areas in medicine, it may be sufficient to maintain high image quality only in the region of interest, i.e., in diagnostically important regions. This paper discusses a hybrid technique for lossless compression in the region of interest, with high-rate lossy compression in other regions. Such techniques are of great interest in telemedicine or medical imaging applications with large storage requirements. In doing so, we are able to achieve a relatively high compression ratio for the MRI images, while preserving the lossless contents of important regions. An application to MRI Brain imaging with the special interest on tumor are presented.
\end{abstract}

\section{KEYWORDS}

Lossless compression, Lossy compression, Region of Interest, and Telemedicine.

\section{INTRODUCTION}

Medical imaging has a great impact on diagnosis of diseases and preparation to surgery. On the other hand, the storage and transmission are an important dilemma due to enormous size of medical imaging data. For example, each slice of CT abdomen images has 512 by 512 of 16 bits, and the data set consists of 200 to 400 images leading to $150 \mathrm{MB}$ of data in average. An efficient compression of the medical data can solve the storage and transmission problem[1].

Current compression schemes bring great compression rates if loss of quality is affordable. Medicine can not afford deficiency in diagnostically important regions ('Region of Interest'). An approach that brings high compression rate with good quality in region of interest (ROI) are required. A hybrid coding scheme seems to be the only solution to this twofold problem. In other words, two different compression schemes should be used for ROI and non-ROI. The

\footnotetext{
${ }^{*}$ Egyptian Armed Forces

Cairo University
} 
general theme is to preserve quality in diagnostically critical regions, while encoding the other regions so that the viewer can observe the position of the critical regions in the original image. Therefore, a very lossy compression scheme is suitable for non-ROI regions to give a global picture to the user while a lossless compression scheme is necessary for ROI regions. After the evolution of digital imaging techniques, many researchers have attempted to apply compression methods to medical data. The initial emphasis was on information preserving methods. Scan pixel difference was researched by Takaya et al in [2]. Assche et a.l exploit the inter-frame redundancy in [3]. Linear predictive coding schemes were investigated in [4]. The lossless compression studies have all resulted in low compression rate. Transform coding schemes such as Principal Component Analysis (PCA) and Discrete Cosine Transform (DCT) were applied in [5],[9] and [10] to get better rates. In order to achieve higher compression rates without detracting from quality, region of interest based methods were investigated in the subsequent years. In [4], a ROI-DCT algorithm that uses more DCT coefficients in ROI, was proposed. Cosman et al. used a subband compression scheme in [6] and [7] for application to mammography. In [8], 3-D wavelet compression was investigated.

The overall goal of this paper is to find a combination of methods, which achieves the highest overall compression performance. The proposed approach is based on ROI, i.e. to segment the image into several regions according to their spatial characteristics, and then compress them separately with different methods.

After evaluation of different lossless and lossy compression techniques, the obtained results suggest that JPEG-LS coding [11] (for lossless mode), and Wavelet-based compression [12] (for lossy mode) are more suitable than other methods for the compression of MRI brain images.

JPEG-LS, the new standard for lossless JPEG compression is based on a variation of the low complexity lossless compression method (LOCO-I). Wavelet-based compression, on the other hand, has several desired features if it is adopted for radiological image compression. Wavelet-based image processing methods in general have gained much attention in the biomedical imaging community. Applications range from compression of medical images, CT reconstruction; local tomography, Wavelet denoising (MRI, Ultrasound), Wavelet-based feature extraction; texture and statistical descriptors, Medical image enhancement (e.g., fluoroscopy and mammography), Analysis of functional images of the brain (positron emission tomography (PET), functional MRI (fMRI)), and Wavelet-encoded MRI. Image compression methods that use wavelet transforms (which are based on multiresolution analysis) have been successful in providing high compression ratios while marinating good image quality, and have proven to be serious competitors to DCT-based or Fractal-based compression schemes. In several papers the suitability of different lossy compression schemes with respect to medical images has been investigated thoroughly. In accordance to the results for general image types it has been found that Wavelet-based compression schemes offer the best rate-distortion performance of all coders considered. The performance of the two compression schemes with a number of lossless and lossy compression schemes including the Said-Pearlman (S+P) transform [13], JPEG [14], JPEG2000 [15], the Burrows-Wheeler transform (BWT) [16], the Lempel-Ziv algorithms [17], and many other lossless and lossy compression techniques were compared.

This paper presents and compares two methods for the compression of medical images that allow an image to be selectively compressed. These techniques offer different functionality (e.g. different types of progressive transmission capability) and show different compression performance. 
The proposed analysis is applied on two datasets MRI brain images of 10 slices each, with the special concern on tumor (Meningioma tumor). The first step of a ROI-based system is segmentation. In the selected applications, the tumor is semi-automatically segmented using Edge-based segmentation followed by a sequence of 2-D morphological image processing operations. Once the ROI is segmented in each slice, a hybrid compression scheme is used for coding the images.

\section{ROI BASED SYSTEM}

This section describes a hybrid compression system for lossless compression of ROI in MRI Brain images. The first stage is the segmentation of ROI. Here, a semi-automated segmentation method is proposed that utilizes an Edge-based segmentation followed by a sequence of 2-D morphological image processing operations as described in section-3. Once the ROI is segmented in each slice, a hybrid compression scheme is used for coding the images. The details of this compression stage are described in section-4 and section-5 respectively. In this study, it is recommended not to discard the non-ROI, but rather to highly compress it by Wavelet-based compression.

\section{SEGMENTATION OF ROI}

The proposed segmentation algorithm relies on Edge-based segmentation followed by a sequence of 2-D morphological image processing operations. The first stage of segmentation is the edge detection algorithm. A practical value to the proposed system is added by designing an edge detection algorithm that produces reliable, robust, and smooth edges [18]. The second stage is a sequence of 2-D mathematical morphology, a branch of science that is built upon set theory with many application areas in image processing. It includes generation of mapping for each pixel according to the pixel's local neighborhood, where, researchers have used this technique to segment biomedical images [19] [20]. In other words, it is composed of a series of dilation operations. Figure 1 through figure 3 show the ROI in the entire images and the segmented ROI alone. Each pixel in the segmented ROI is coded in a lossless manner, while the rest is lossy compressed.

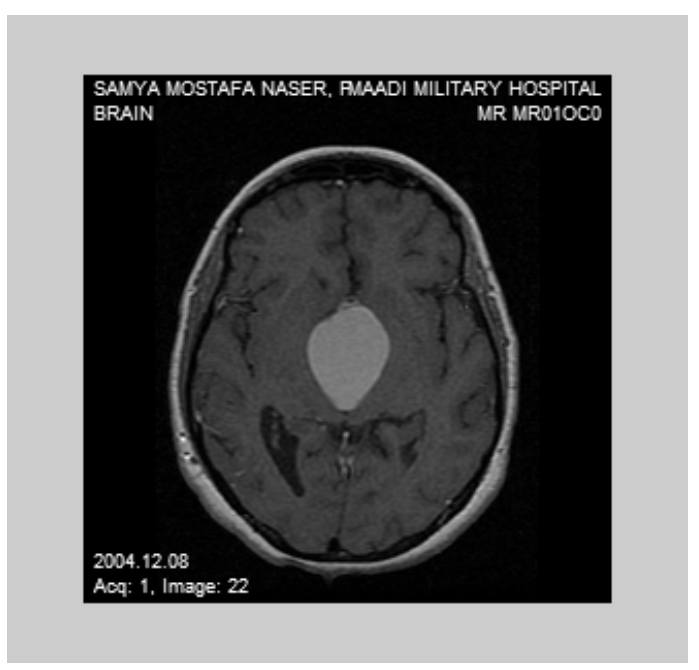

(a)

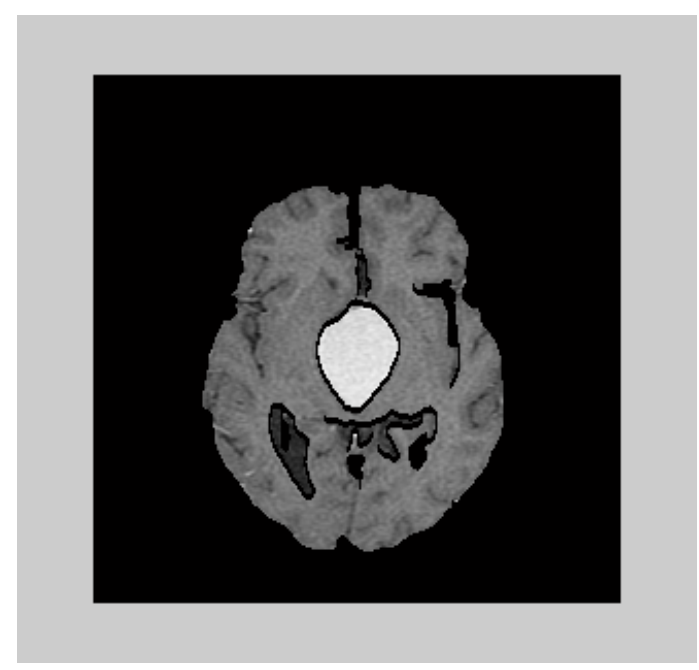

(b)

Fig. 1: (a) ROI together with the other organs. (b) ROI alone. 


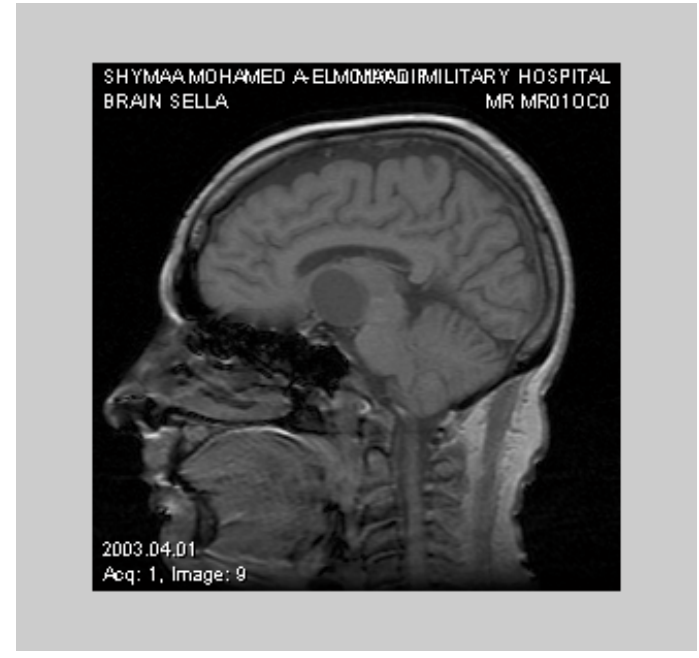

(a)

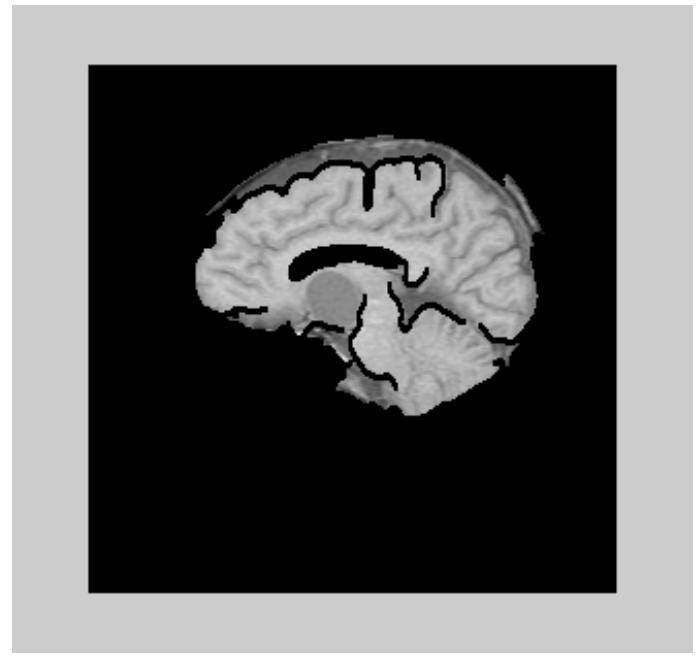

(b)

Fig. 2: (a) ROI together with the other organs. (b) ROI alone.

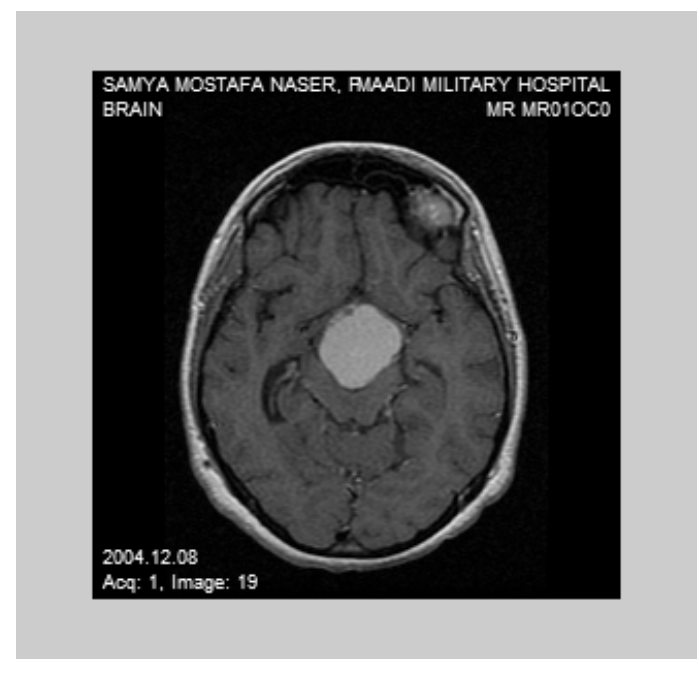

(a)

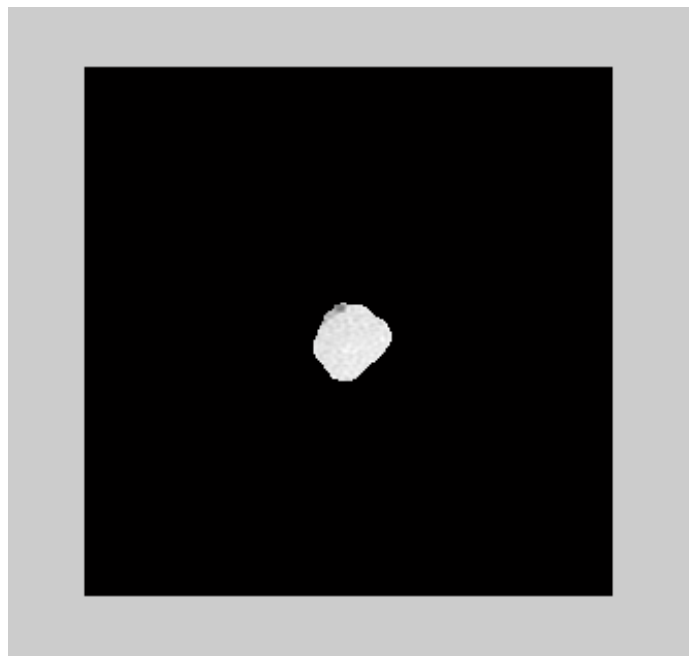

(b)

Fig. 3: (a) ROI together with the other organs. (b) ROI alone.

\section{DIRECT ROI CODING}

The image data to be compressed consist of different components: the ROI and the remainder. The ROI data consist of one or more arbitrarily shaped parts of the image to be compressed in lossless mode; the remainder (again with arbitrary shape) is to be compressed in a lossy mode. The ROI data consist of two parts - the actual image data (grey values, texture information) and information about the shape and position of the region. The ROI data is "cut out" of the image and compressed in lossless mode. Subsequently, the reminder of the image is compressed in lossy manner.

\section{RESIDUAL ROI CODING}

As in the previous setting, the image data to be compressed consist of different components: the ROI and the remainder. But in contrast to direct ROI coding, the entire images are 
compressed in lossy mode with the target bit rate first. Subsequently, the 'residual ROI' is computed. This is the difference between the lossy compressed ROI and the corresponding original image data in the ROI for each slice. The residual ROI is then stored in lossless mode. Consequently, the ROI data may be restored without degradation. After decompressing the lossy compressed data, the residual ROI is simply added to the regions defined as being ROI.

\section{COMPARISON OF THE ALGORITHMS}

Different behavior concerning compression performance and functionality are to be expected of the different algorithms considered. The residual ROI coding algorithm may be expected to offer better compression performance due to the following reason: the data to be stored in lossless mode is of residual type. In contrast to the direct method where plain image data is encoded. Residual type data shows a lower entropy values which is the reason for the expected better encoding behavior.

On the other hand, direct ROI coding offers different functionality as compared to the residual ROI coding: the ROI may be displayed independently without decompressing the image reminder in the case of direct ROI coding only. When using residual ROI coding the entire image needs to be decompressed first, subsequently the ROI may be reconstructed. Therefore, in all kinds of applications where compression performance is crucial residual ROI coding is the method to choose. If progressivity according to the importance of the image content is required (i.e. to display lossless coded ROI data first and the image reminder subsequently) this can be achieved by using direct ROI coding.

\section{ROI-BASED COMPRESSION PERFORMANCE EVALUATION}

The following components are used for the algorithms in our experiments:

- Lossless compression: we apply the JPEG-LS lossless compression.

- Lossy compression: we use the Wavelet-based lossy compression.

- ROI-based compression: The ROI data is compressed in lossless mode where, the reminder of the image is compressed in lossy manner.

We have chosen different ROI size in order to present results of a critical application setting, if small ROI are considered; the achievable compression ratios are much higher. The algorithms were tested on two datasets MRI brain images of 10 slices each, with 8 bits/pixel (bpp) and $256 \times 256$ pixels each.

Before we give our results on the ROI based scheme, we evaluate the lossless JPEG-LS and the Wavelet-based lossy compression, table 1 summarizes the objective and subjective evaluation of the methods. Note that the lossless and lossy compression algorithms applied to the original images without considering the ROI.

Table 1: Objective and subjective evaluation of JPEG-LS and

Wavelet-based compression.

\begin{tabular}{||c||c|c|c|c||}
\hline \multirow{2}{*}{ Image Name } & $\begin{array}{c}\text { JPEG-LS } \\
\text { Compression }\end{array}$ & \multicolumn{3}{|c|}{ Wavelet-based Compression } \\
\cline { 2 - 5 } & CR & Critical CR & $\begin{array}{c}\text { PSNR } \\
\text { (dB) }\end{array}$ & $\begin{array}{c}\text { Visual } \\
\text { Inspection }\end{array}$ \\
\hline \hline 001 T1 & 2.08 & 14.75 & 30.72 & Good \\
\hline
\end{tabular}




\begin{tabular}{||c||c||c|c|c||}
\hline $002 \_\mathrm{T} 1$ & 2.22 & 13.97 & 32.6 & Accepted \\
\hline $003 \_\mathrm{T} 1$ & 2.15 & 13.42 & 32.2 & Accepted \\
\hline $004 \_\mathrm{T} 1$ & 2.4 & 16.4 & 33.6 & Accepted \\
\hline $005 \_\mathrm{T} 1$ & 2.4 & 14.04 & 34.88 & Accepted \\
\hline $006 \_\mathrm{T} 1$ & 2.23 & 12.54 & 34.17 & Accepted \\
\hline 007 T1 & 2.2 & 14.1 & 32.65 & Accepted \\
\hline $008 \_\mathrm{T} 1$ & 2.4 & 12.8 & 35.73 & Good \\
\hline $009 \_\mathrm{T} 1$ & 2 & 11.36 & 33.86 & Accepted \\
\hline $0010 \_\mathrm{T} 1$ & 2.42 & 14.6 & 35.2 & Accepted \\
\hline \hline $001 \_\mathrm{T} 2$ & 2.35 & 15.6 & 32.4 & Good \\
\hline $002 \_\mathrm{T} 2$ & 2.41 & 15.4 & 32.58 & Accepted \\
\hline $003 \_\mathrm{T} 2$ & 2.17 & 14.5 & 31.76 & Accepted \\
\hline $004 \_\mathrm{T} 2$ & 2.4 & 14.7 & 33.19 & Accepted \\
\hline $005 \_\mathrm{T} 2$ & 2.4 & 13.94 & 33.06 & Good \\
\hline $006 \_\mathrm{T} 2$ & 2.45 & 14.3 & 33.27 & Good \\
\hline $007 \_\mathrm{T} 2$ & 2.5 & 15.4 & 32.8 & Accepted \\
\hline $008 \_\mathrm{T} 2$ & 2.52 & 16.45 & 33.23 & Good \\
\hline $009 \_\mathrm{T} 2$ & 2.36 & 14.17 & 32.33 & Good \\
\hline $0010 \_\mathrm{T} 2$ & 2.45 & 15.8 & 32.98 & Good \\
\hline \hline
\end{tabular}

This table shows that, the MRI brain images can be losslessy compressed at relatively low compression ratios, ranging from $2: 1$ to over 2.5:1. It is clear that the MRI brain images can be lossy compressed at relatively high compression ratios. This is indicated in the column of "Critical Ratio" as identified by the expert radiologists. As expected, the critical ratio of compression is different from one image to another, ranging from 11:1 to over 16:1. An interesting observation of the PSNRs is that the PSNRs are fall within a range between $30 \mathrm{~dB}$ to $35 \mathrm{~dB}$, even though the compression ratios change significantly from one case to another.

Next, we would like to give theoretical entropy values of the ROI regions in both cases, i.e. plain image data (direct ROI), and residual type data (residual ROI). Then, we apply our ROIbased hybrid compression methods to the two datasets of 20 slices. JPEG-LS lossless compression to the ROI region in the case of direct ROI and to residual ROI region in the case of residual ROI, and the Wavelet-based lossy compression, with the target bit rate determined from table 1 , to the reminder of the images. In the ROI-based scheme, the compressed image obviously consists of both lossless and lossy coded parts. Using the combined algorithms, the output results are summarized in table 2 .

Table 2: Results of ROI-based compression scheme

\begin{tabular}{|c|c|c|c|c|c|c|c|c|c|}
\hline \multirow[b]{2}{*}{$\begin{array}{l}\text { Image } \\
\text { Name }\end{array}$} & \multirow{2}{*}{$\begin{array}{l}\text { ROI } \\
\text { Size } \\
(\%) \\
\end{array}$} & \multicolumn{4}{|c|}{ Direct-ROI } & \multicolumn{4}{|c|}{ Residual-ROI } \\
\hline & & $\begin{array}{c}\text { ROI } \\
\text { Entropy } \\
\end{array}$ & CR & $\begin{array}{l}\text { PSNR } \\
\text { (dB) }\end{array}$ & $\begin{array}{c}\text { Visual } \\
\text { Inspection }\end{array}$ & $\begin{array}{c}\text { R. ROI } \\
\text { Entropy }\end{array}$ & CR & $\begin{array}{l}\text { PSNR } \\
\text { (dB) }\end{array}$ & $\begin{array}{c}\text { Visual } \\
\text { Inspection }\end{array}$ \\
\hline "001_T1 & 32.16 & 3.22 & 4.4 & 32.6 & Excellent & 2.88 & 5.2 & 32.75 & Excellent \\
\hline $002 \mathrm{~T} 1$ & 0.92 & 0.12 & 12.3 & 32.6 & Good & 0.11 & 12.6 & 32.64 & Good \\
\hline $003 \mathrm{~T} 1$ & 2.2 & 0.26 & 10.46 & 32.27 & Good & 0.24 & 11.1 & 32.3 & Good \\
\hline 004 T1 & 28.02 & 2.33 & 4.5 & 34.68 & Excellent & 1.95 & 4.8 & 35 & Excellent \\
\hline $005 \mathrm{~T} 1$ & 1.7 & 0.19 & 11.67 & 34.93 & Good & 0. & 12.14 & 4.95 & Good \\
\hline 006 T1 & 1.54 & 0.18 & 10.6 & 34.22 & Good & 0.1 & 11.04 & 34.24 & Good \\
\hline $007 \mathrm{~T} 1$ & 1.2 & 0.15 & 12.1 & 32.69 & Good & 0.13 & 12.53 & 32.71 & Good \\
\hline 008 T1 & 27.8 & 2.32 & 3.9 & 37.15 & Excellent & 1.86 & 4.4 & 37.45 & Excellent \\
\hline 009_T1 & 21.3 & 1.98 & 4.34 & 34.4 & Excellent & 1.53 & 5.01 & 34.73 & Excellent \\
\hline 0010_T1 & 19.7 & 1.76 & 5.4 & 36.48 & Excellent & 1.48 & 5.7 & 36.65 & Excellent \\
\hline
\end{tabular}




\begin{tabular}{|c|c|c|c|c|c|c|c|c|c|}
\hline 001 T2 & 6.53 & 0.76 & 8.51 & 32.76 & Excellent & 0.64 & 9.27 & 32.9 & Excellent \\
\hline $002 \mathrm{~T} 2$ & 8.0 & 0.92 & 8.36 & 32.93 & Excellent & 0.73 & 8.9 & 33.02 & Excellent \\
\hline 003 T2 & 0.7 & 0.1 & 13.0 & 31.75 & Good & 0.08 & 13.3 & 31.8 & Good \\
\hline $004 \mathrm{~T} 2$ & 16.9 & 1.73 & 4.93 & 34.2 & Excellent & 1.37 & 5.8 & 34.4 & Excellent \\
\hline $005 \mathrm{~T} 2$ & 1.62 & 0.21 & 11.34 & 33.15 & Good & 0.18 & 11.94 & 33.17 & Good \\
\hline 006_T2 & 30.2 & 2.9 & 3.83 & 35.72 & Excellent & 2.2 & 4.1 & 35.94 & Excellent \\
\hline 007 T2 & 2.1 & 0.24 & 12.36 & 32.84 & Good & 0.21 & 12.9 & 32.9 & Good \\
\hline 008_T2 & 25.37 & 2.46 & 4.71 & 35.28 & Excellent & 1.93 & 4.9 & 35.6 & Excellent \\
\hline $009 \mathrm{~T} 2$ & 3.11 & 0.39 & 9.9 & 32.44 & Good & 0.33 & 10.7 & 32.52 & Good \\
\hline $0010 \mathrm{~T} 2$ & 27.55 & 2.7 & 4.23 & 35.0 & Excellent & 2.05 & 4.6 & 35.24 & Excellent \\
\hline
\end{tabular}

Based on these evaluation results, we are able to draw several conclusions:

1. For all considered images, the theoretical entropy of the residual ROI regions is less than the theoretical entropy of the plain ROI regions. This explains the expected better encoding behavior of the residual ROI method over the direct ROI method.

2. As expected, the residual ROI method outperforms the direct ROI method in term of compression ratio. The CRs are fall within a range between 3.9:1 to 12.1:1 for direct ROI method and 4.4:1 to 12.53:1 for residual ROI method.

3. We may also notice a PSNR gain of the residual ROI coding over the direct ROI coding of about $0.3 \mathrm{~dB}$.

4. ROI-based compression methods are better than Wavelet-based lossy compression in terms of both objective measure of PSNR and subjective measure of expert visual inspections, since the ROI region in both methods has high quality of image and the quality in the other regions is degraded.

5. On the other hand, the Wavelet-based lossy compression is consistently better than ROI-based compression in terms compression ratio.

6. ROI-based compression methods show a considerably higher compression ratio as compared to the JPEG-LS lossless compression scheme, while critical information is preserved.

7. The visual perception of the two methods is the same for all images.

8. The ROI size is different from one image to another, ranging from $1 \%$ to over $32 \%$.

9. The efficiency of the methods is inversely proportional to the portion of the ROI in the image. The smaller the portion of ROI in the image, the better is the resulting compression rate.

In order to judge the performances of the applied methods, figure 4 through figure 6 show the error image after ROI-based decompression, and Lossy decompression. The error appears as white pixels.

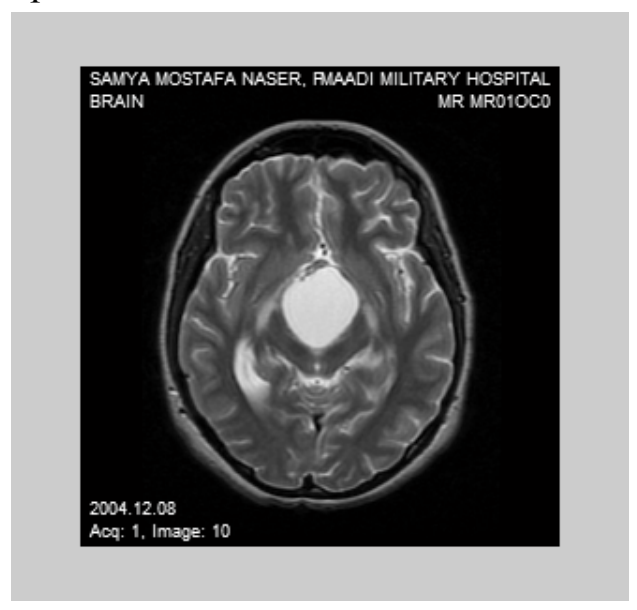

(a)

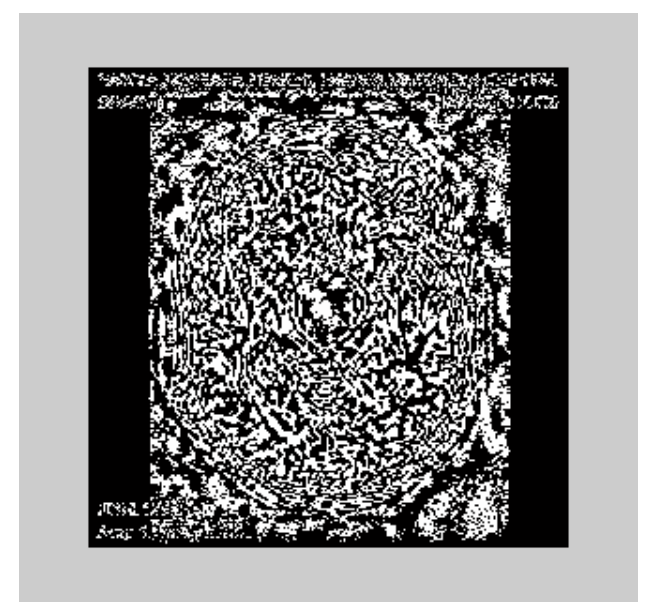

(b) 
Proceedings of the $5^{\text {th }}$ ICEENG Conference, 16-18 May, 2006 


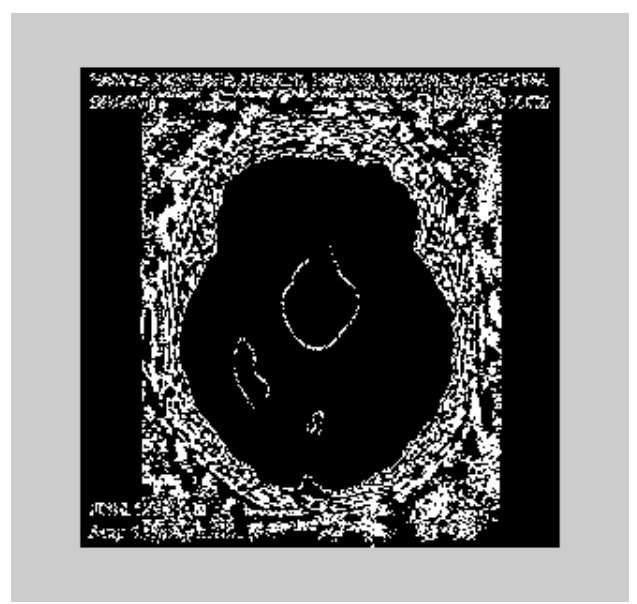

(c)

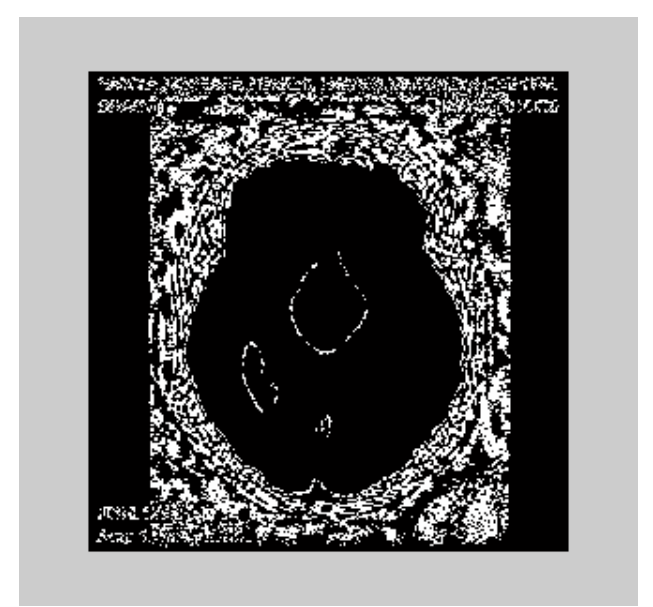

(d)

Fig 4: (a) Original image. (b) Error image after Wavelet decompression.

(c) Error image after Direct ROI decompression. (d) Error image after Residual ROI decompression.

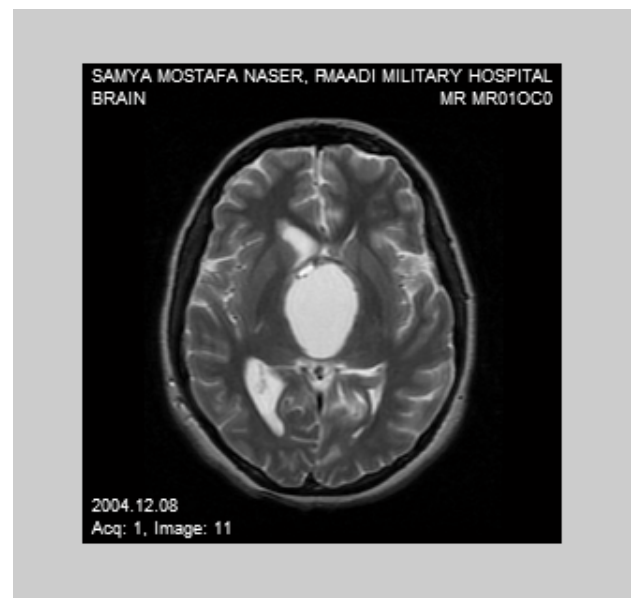

(a)

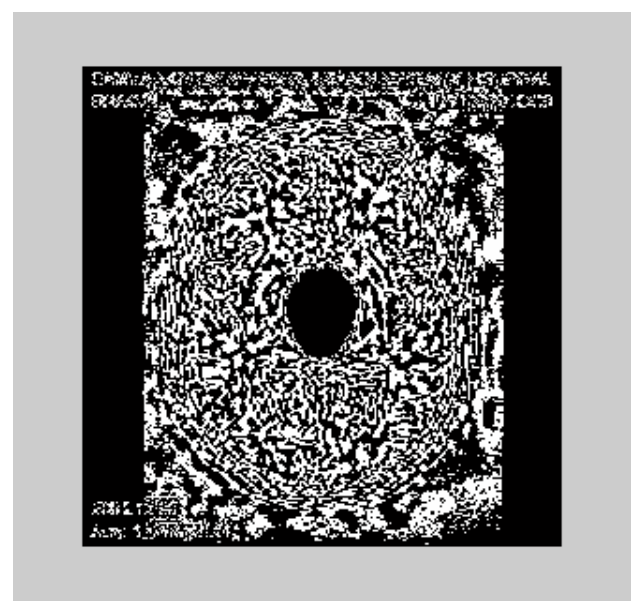

(c)

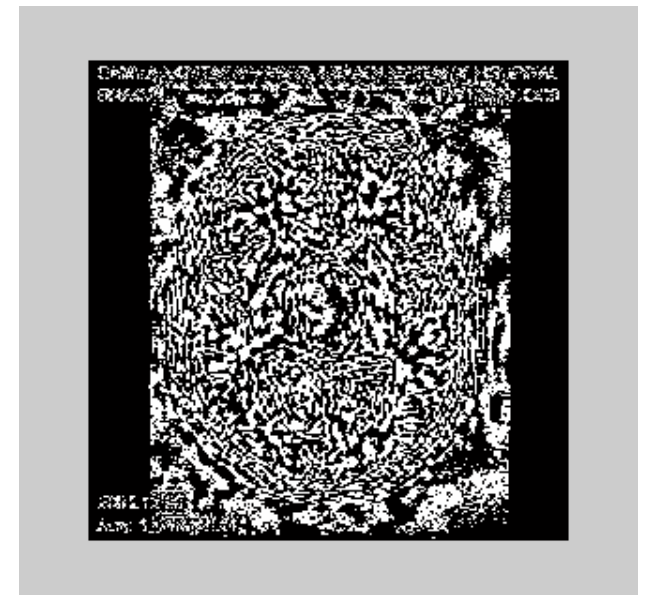

(b)

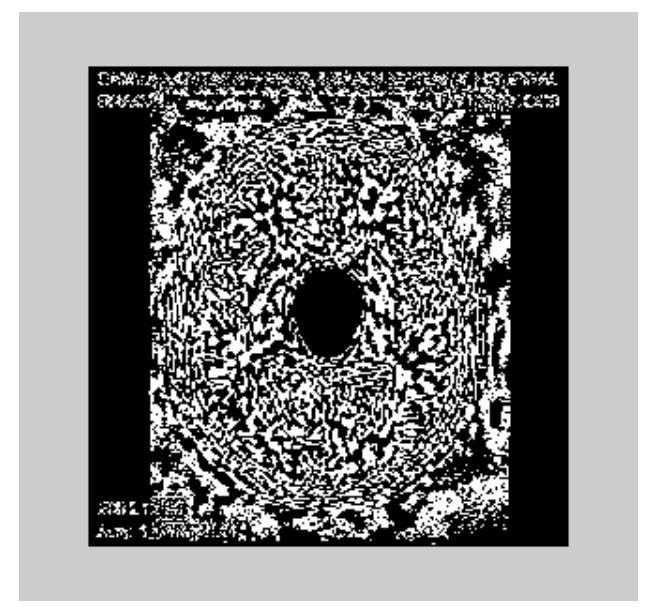

(d)

Fig 5: (a) Original image. (b) Error image after Wavelet decompression.

(c) Error image after Direct ROI decompression. (d) Error image after Residual ROI decompression. 


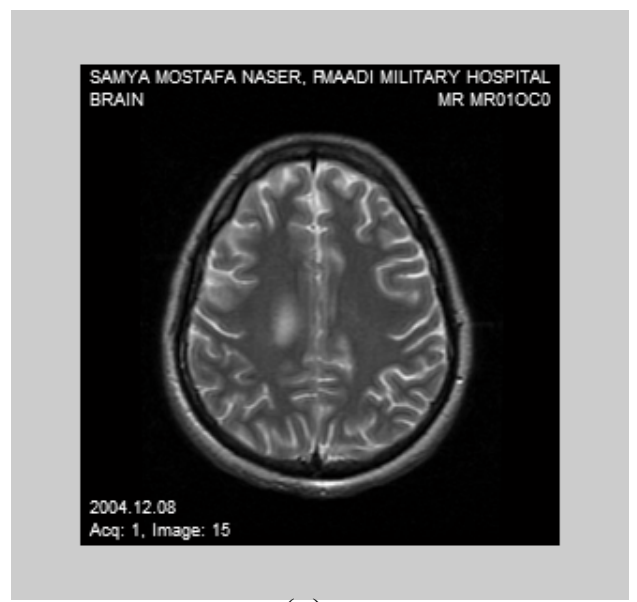

(a)

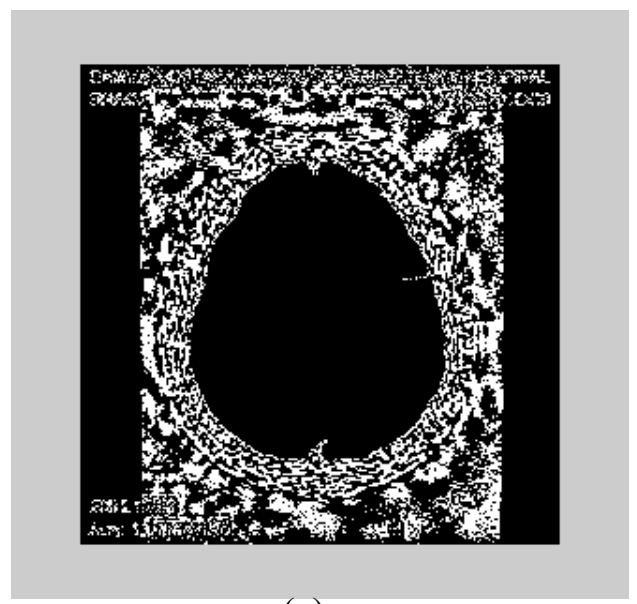

(c)

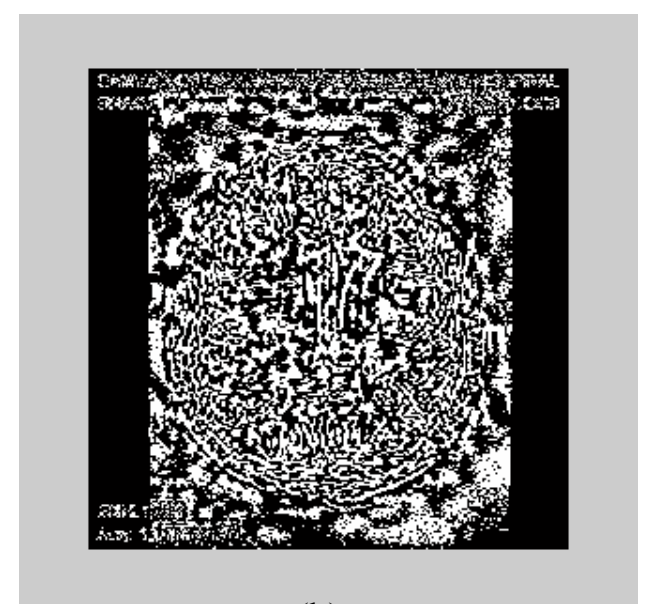

(b)

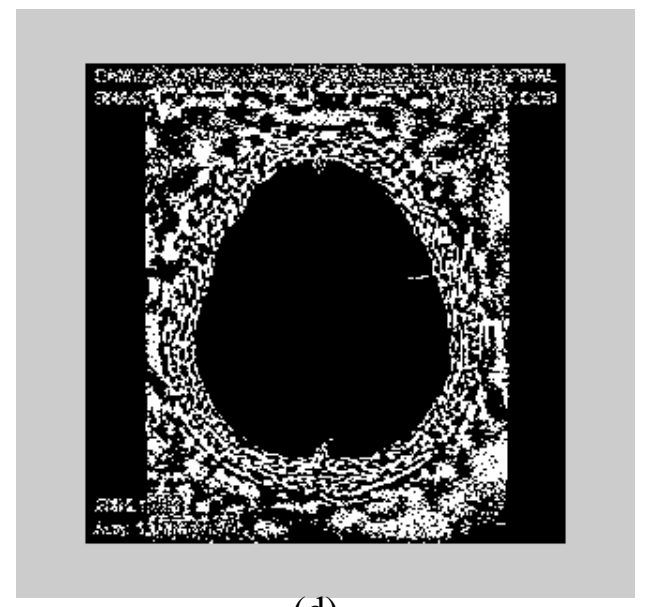

(d)

Fig 6: (a) Original image. (b) Error image after Wavelet decompression. (c) Error image after Direct ROI decompression. (d) Error image after Residual ROI decompression.

Figure 4 through figure 6 show that, the algorithms are accurate, since there is no degradation of diagnostic quality in ROI.

Summarizing, we may state that the theoretically predicted properties of the different ROI image compression algorithms are fully confirmed in these experimental results.

\section{CONCLUSION}

There are quite a few applications in which one would like certain portions of an image to be encoded with higher quality than other portions. Medical images are among the few where people argue that higher quality should actually mean lossless quality. (Indeed, many physicians and scientists have argued that medical images must always be compressed losslessly everywhere.) However, we propose that regionally lossless compression methods have the potential for widespread medical acceptance, since they are responsive to the nature of medical images (spatially varying importance levels and statistical characteristics) and to the needs of the radiologist (guaranteed accuracy for diagnostically important regions, no ambiguity about what portions are reliable). 
In this study, we present a hybrid scheme that uses lossless compression in the region of interest, and very high-rate, lossy compression in the other regions. The main contribution of this paper is the design of an edge detection algorithm that produces reliable, robust, and smooth edges. After surveying common compression schemes, we have chosen JPEG-LS coding (for lossless mode), and Wavelet-based (for lossy mode). The proposed method is applied on MRI brain images with the tumor as ROI. The results depended on the size of the ROI.

We observe that for a ROI-based compression application the so-called residual ROI coding approach turns out to be superior with respect to compression performance and rate-distortion performance Therefore, in all kinds of applications where compression performance and ratedistortion performance are crucial residual ROI coding is the method to choose. If progressivity according to the importance of the image content is required (i.e. to display lossless coded ROI data first and the image reminder subsequently) this can be achieved by using direct ROI coding. The same is true of course for random access to the ROI data without considering the image reminder at all.

Continuations of this project might include comparisons of methods for multiple ROI regions, the design of lossy compression schemes in ROI, and a clinical case study with radiologists to observe the effect of lossy compression on diagnostic performance.

\section{REFERENCES}

[1]S.B.Gokturk,C.Tomasi,B.Girod,and C.Beaulieu, "Medical image compression based on region of interest, with application to colon CT images," Engineering in Medicine and Biology Society (EMBS). Proceedings of the 23rd Annual International Conference of the IEEE, vol.3, pp: 2453 -2456, 2001.

[2] K. Takaya, and C.G. Tannous, "Information preserved guided scan pixel difference coding for medical images," WESCANEX. Communications, Power, and Computing. Conference Proceedings, IEEE Volume: 1, pp: 238 -243, 1995.

[3] S.Van Assche,, D. De Rycke, W. Philips, and I. Lemahieu, "Exploiting interframe redundancies in the lossless compression of 3D medical images," Data Compression Conference, pp: 575, 2000.

[4] Jian-Hong Hu, Yao Wang, and P.T. Cahill, "Multispectral code excited linear prediction coding and its application in magnetic resonance images," IEEE Transactions on Image Processing, Volume: 6 11, pp: 1555 -1566, Nov. 1997.

[5] A.Vlaciu, S. Lungu, N. Crisan, and S.Persa. "New compression techniques for storage and transmission of 2-D and 3-D medical images," In Advanced Image and Video Communications and Storage Technologies, volume 2451, pages 370-7, Amsterdam, Netherlands, March 1995.

[6] R.M. Gray, R.A. Olshen, D. Ikeda, P.C. Cosman, S. Perlmutter, C. Nash, and K. Perlmutter, "Evaluating quality and utility in digital mammography," Proceedings of International Conference on Image Processing, Volume: 2 , pp: 5 -8, 1995.

[7] P.C. Cosman, R.M. Gray, and R.A. Olshen, "Evaluating quality of compressed medical images: SNR, subjective rating, and diagnostic accuracy," Proceedings of the IEEE, Volume: 82 6, pp: 919-932, June 1994. 
[8] A. Baskurt, F. Peyrin, H. Benoit-Cattin, and R. Goutte, "Coding of medical images using 3D wavelet decompositions," IEEE International Conference on Acoustics, Speech, and Signal Processing, ICASSP-93, Volume: 5, Page(s): 562 -565, 1993.

[9] J.S. Taur, and C.W. Tao, "Medical Image Compression using Principal Component Analysis," International Conference on Image Processing, Volume: 1, Page(s): 903 -906, 1996.

[10] M. Yoshioka, and S. Omatu, "Image Compression by nonlinear principal component analysis," IEEE Conference on Emerging Technologies and Factory Automation, EFTA '96,Volume: 2 , pp: 704 -706, 1996.

[11] Marcelo J. Weinberger, Gadiel Seroussi, and Guillermo Sapiro,” The LOCO-I Lossless Image Compression Algorithm: Principles and Standardization into JPEG-LS" IEEE transactions on image processing, Vol. 9, No. 8, August 2000.

[12] Michel U., Akram A., Andrew L., "Guest Editorial: Wavelets in Medical Imaging" IEEE Transactions on Medical Imaging, Vol. 22, No. 3 285-288 March 2003.

[13] A. Said and W. A. Pearlman, "An image Multiresolution Representation for Lossless and Lossy Compression,” IEEE Trans. Image Processing, pp. 1303-1310, 1996.

[14] W. B. Pennebaker and J. L. Mitchell, "JPEG: Still Image Data Compression Standard." New York: Van Nostrand Reinhold, 1992.

[15] D. Santa-Cruz, T. Ebrahimi, J. Askelf, M. Larsson and C. A. Christopoulos, "JPEG 2000 still image coding versus other standards.” ISO/IEC JTC1/SC29/WG1 N1816 July 2000.

[16] M. Burrows and D. J. Wheeler. "A block-sorting lossless data compression algorithm." SRC Research Report 124, Digital Equipment Corporation, Palo Alto, California, May 10, 1994.

[17] J. Ziv and A. Lempel, "A universal algorithm for sequential data compression," IEEE Trans. Inform. Theory, vol. IT-23, pp. 337-343, May 1977.

[18] H. Aboelsoud M., Abdalla S. Ahmed, M. E. Gadalla, and A. S. Ragab, Systems and Biomedical Engineering Department, Cairo University, Egypt. "Edge Detection From The Third Derivative," Submitted for publication.

[19] R.Vogt, "Precise Extraction of Bones from CT Scans," Advances in Mathematical Morphology, Volume 2, 1982.

[20] J.Serra, "Image Analysis and Mathematical Morphology," Academic Press, New York, N.Y., 1982. 
